\title{
Commitment in sequential auctioning: advance listings and threshold prices
}

\author{
Robert Zeithammer
}

February 7, 2008

\begin{abstract}
This paper models sequential auctioning of two perfect substitutes by a strategic seller, who learns about demand from the first-auction price. The seller holds the second auction only when the remaining demand is strong enough to cover her opportunity cost. Bidding in anticipation of such a contingent future auction is characterized, including a sufficient condition for existence of an invertible (increasing symmetric pure-strategy) bidding equilibrium that facilitates the seller's learning. A unique invertible bidding equilibrium exists for the Dutch auction format, but only when the second auction is sufficiently discounted by the bidders. In the equilibrium, highvaluation bidders shade their bids down as if the second auction were guaranteed. To counter such strategic bidding, the seller would value ex-ante commitment to hold the second auction less often. Three forms of such commitment are analyzed: commitment to list future auctions in advance, commitment to not hold the second auction unless the first price exceeds a publicly announced threshold, and commitment to a reserve-price in the second auction.
\end{abstract}

Keywords: Auctions, Game-theory, Optimal selling, Commitment, Non-commitment dynamic pricing.

I would like to thank Georgios Katsenos, Thomas Jeitschko, Miguel Villas-Boas, George Deltas, and an anonymous referee for thorough and insightful feedback. Address: Anderson School of Management, UCLA, 110 Westwood Plaza, Los Angeles, CA 90095. email : rzeitham@alum.mit.edu. Phone : (310)-825-1862 


\section{Introduction}

Unit-demand goods are frequently auctioned in a sequence of standard auctions, one unit at a time. On eBay, dealers and individuals sell unit-demand consumer durables, such as cars or electronics, using sequences of English auctions. Flower growers in the Netherlands and elsewhere sell carts of flowers using sequences of Dutch auctions. Finally, governments and large firms use sequences of first-price sealed-bid auctions to sell procurement contracts - unit demand goods to capacity-constrained firms. A seller of such goods facing persistent buyers has the opportunity to learn about demand from the early auctions and adapt her selling strategy going forward. For example, she may be better off not producing additional units when the remaining demand is weak. To accommodate such a selling strategy, most auctioneers give sellers the right to withdraw future lots from the auction. While clearly beneficial, learning more about remaining demand also comes with a cost of lower revenue in the early auctions because high-value bidders reduce their bids in response to a learning seller. They know that losing an early auction to an even higher competitor guarantees a high-demand signal that will entice the seller to offer another unit. Therefore, the high-value bidders can bid conservatively in the early auctions, knowing that there will be another chance to buy the good should they lose. This paper analyzes the resulting tradeoff between benefits and costs of learning, and finds that the seller can profit from various forms of commitment to reduce future supply.

A concrete example is useful to both show an application of the theory, and to illustrate the different commitment strategies under investigation. Consider a flower grower selling carts of the same flowers to florists, with the auction format exogenously fixed to a Dutch auction (as in the world's largest Aalsmer flower-auction house in the Netherlands). The grower has a cart of flowers to sell today, and she can produce another cart tomorrow at some publicly known cost. When there is a unique increasing symmetric pure-strategy bidding equilibrium in today's auction, today's price reveals the valuation of the highest-valuation florist, who subsequently exits the game because each florist only wants to buy one cart (unit-demand). The winner's revealed valuation is an upper bound on the valuations among the remaining bidders, so a noncommitment grower (who makes decisions one unit at a time) will produce and sell the second cart when her cost of producing it is less than the expected profit implied by the upper bound on valuations. Anticipating that decision in the first auction, florists with high-enough valuations 
can take the second sale for granted and bid as if there were two carts for sale. In equilibrium, these high-valuation florists therefore bid less aggressively than in an isolated single-cart auction - a phenomenon called bid-shading here.

Subgame perfection may force the non-commitment grower to produce and sell the second cart of flowers even when her bid-shading revenue-loss on the first cart exceeds the expected profits from the second cart. Under those market conditions, the grower would clearly benefit from some form of ex-ante commitment to reduce future production. The simplest such commitment is available to a seller who foregoes learning completely and always lists future auctions in advance: when she does not list a future auction, the bidders believe that there will not be one. Advance listing can be credible when there is a third-party auctioneer who requires advance notice (as effectively does eBay), or when such advance notice is a necessary feature of the market (as in procurement). ${ }^{1}$ This paper provides a general characterization of seller preference for advance listing over non-commitment. When the distribution of valuations is uniform, the problem is tractable in that a unique cost-cutoff emerges above which the seller prefers to list in advance (keeping the number of bidders constant). The cutoff rises with the number of bidders, so increased demand-side competition makes advance listing less desirable.

The complete loss of learning implied by advance listing is not a necessary feature of optimal selling when the grower can commit to second-period strategies contingent on the firstauction price. Instead of making the decision about the second cart in the beginning of the game, the grower may be able to announce a threshold price - minimum first-cart price above which she will produce and sell the second cart. This may be credible when the seller is an agent who faces piecemeal incentives: for example, political pressure may force the government to cancel future infrastructure projects if the inaugural project turns out to be too expensive once contractors bid on it. Alternatively, credibility can always be established through reputation. It is immediate that an optimally selected threshold price will outperform both advance listing and non-commitment: First, extremely low or high thresholds replicate profits from advance listing. Second, a threshold slightly above the price that makes the non-commitment seller break even in the second period outperforms non-commitment because it reduces shading without a marginal reduction in profits. This paper analyzes the bidding strategies induced by a threshold selling

\footnotetext{
${ }^{1}$ On eBay, auctions are open for bidding for several days, and so the economically relevant endgame is effectively listed several days in advance. To allow bid-preparation, government auctions of procurement contracts for highway construction are listed at least four weeks in advance (Jofre-Bonet and Pesendorfer 2003).
} 
strategy, and finds that there is a unique increasing first-period bidding strategy if and only if the second period payoffs are sufficiently discounted. Therefore, informativeness of the first-period bids cannot be taken for granted. A general characterization of the optimal threshold price is derived, together with a closed-form result for the uniform distribution.

The threshold price strategy increases profits by convincing low-valuation florists that they should not expect a second cart of flowers on the margin. These florists then do not shade their bids in the first auction, in turn increasing competition for the high-value florists who also end up bidding more thanks to Dutch-auction incentives. Another way to convince low-valuation florists not to expect a second cart would be to pre-commit to a reserve price for the second cart in the beginning of the game. Credibility of a reserve price in the second period requires additional commitment to be credible, namely commitment not to re-auction unsold units (McAfee and Vincent 1997). When the seller does have this additional commitment, an interesting equivalence emerges: the second-unit reserve optimal for the entire sequential-auction profit is the same as the optimal reserve for selling only the second unit to the same group of bidders. In other words, the incentive to reduce first-period bid-shading by raising the secondperiod reserve is perfectly balanced by the incentive to accommodate the weaker second-period demand arising from the highest bidder exiting the market in the end of the first period.

The paper is organized as follows: After a brief literature review in Section 2, Section 3 defines the model, and derives the equilibrium strategies under the baseline assumption that second-period reserve prices are weak (production cost needs to be sunk before the second auction, seller cannot commit not to re-auction). Section 4 then changes these assumptions, considers commitment to a future reserve, and derives the equivalence between a single-auction reserve and pre-commitment reserve in two auctions. Section 5 concludes.

\section{Related literature}

It is well known that bid-shading by all bidders is the best response to an exogenous future auction for a substitute (Milgrom \& Weber 2000, Engelbrecht-Wiggans 1994, Jeitschko 1999, and many others). This paper extends the literature on bidding in sequential auctions by making the existence of the second auction endogenously determined by a strategic seller. The bidders facing a strategic seller shade their bids today only when they expect her to sell another unit after learning about demand from the outcome of the current auction. This is different from Jeitschko 
(1999), who studies an uncertain but still exogenous future sale: while all of Jeitschko's bidders increase their bids in response to the uncertainty, the bidders here increase their bids more when their valuations are low. The most related paper is Zeithammer (2007) who analyzes the relative preference for advance listing over non-commitment in a stylized case of two patient bidders drawn from a discrete Bernoulli distribution. Proposition 4 in this paper generalizes his result to continuous distributions and an arbitrary number of bidders. The strategies available to the seller are expanded to include price-thresholds and future reserve prices. Another closely related paper is Katsenos (2007), who analyzes a complementary model by focusing on a seller with "limited commitment" who can commit not to re-auction unsold units but cannot commit to a future reserve-price. He does not consider advance listing, commitment to a threshold price, or the case when the second-unit production cost needs to be sunk before the second auction. The present setting is also different in that the first item is sold without reserve, so low bidders do not strategically abstain from the first auction as they do in Katsenos (2007).

As a consequence of the strategic selling, increasing symmetric pure-strategy bidding equilibria do not always exist, and so the informativeness (invertibility) of first-period prices cannot be taken for granted. It is well known that such informativeness breaks down when the seller can "ratchet up" the price to extract all remaining buyer surplus (Freixas, Guesnerie, Tirole 1985). Such ratcheting cannot occur in a Dutch auction for unit-demand goods because the valuation of the second-period winner remains hidden in the first-period bids. This logic is the unit-demand good mirror image of the logic that makes the ascending English auction preferable for sequential auctioning of goods with rejuvenating demand in Caillaud \& Mezzetti (2004): Their goods have identical demand in every period, and the ascending English auction keeps the valuation of the second-period winner (the overall highest bidder) hidden. Because of ratcheting, the present model would completely break down if the goods were sold by ascending English or second-price sealed-bid auctions, as explained in detail by Katsenos (2007).

The goods considered here are durable in that bidders are persistent across time-periods and have unit demand over time. The commitment problem of the non-commitment seller is analogous to the commitment problem of a durable-goods monopolist who sets prices (Coase 1972, Stokey 1979). The problem appears even when there is only one unit to sell by auction with a reserve, because the seller will be tempted to lower the reserve price tomorrow in case no bids exceed it today. As a result, the ability to re-auction immediately leads to the Coase 
conjecture - the auction seller cannot credibly use a reserve above her opportunity cost (McAfee \& Vincent 1997). When instantaneous re-auctioning is not possible and the horizon is finite, Skreta (2007) builds on Skreta (2006) to show that the optimal way to sell the single unit is a sequence of auctions with declining reserves. This paper abstracts away from the re-auctioning complications by assuming that re-auctioning is instantaneous. However, the seller still has a commitment problem because there may be more than one unit sold, and she will be tempted to produce and sell the second unit "too often" for overall profit maximization. The analysis contributes to the durable-goods literature by examining a case of a seller who is initially uncertain about demand and subsequently learns about it from the early transactions. As a result of this learning, commitment needs to be sufficiently fine-grained (contingent on observable first-auction outcomes) to prevail over non-commitment. With only a coarse commitment, such as that arising from advance listings, the increased profit from learning can exceed the revenue loss due to lack of commitment, and thus make the non-commitment seller better off. In contrast, the canonical price-setting monopolist of Stokey (1979) faces a perfectly known downwardsloping demand curve and always benefits from commitment not to lower price tomorrow.

\section{Model of sequential auctioning with a threshold price}

There are two periods, 1 and 2. Everyone lives for both periods and discounts second-period outcomes by factor $\delta<1$.

Seller: There is a monopolist risk-neutral seller endowed with one unit of a good in the beginning of the game. In the first period, she sells the first unit in a first-price sealed-bid auction (hereafter 1PSB) without a reserve and observes the first-period price $p_{1}{ }^{2}$ In the second period, she can produce and sell one additional unit at a cost of production $c \geq 0$, where 0 is WLOG the seller's own value of a unit. ${ }^{3}$ The market conventions dictate that the second-period auction must be a 1PSB, possibly with a reserve-price. The production cost $c$ must be sunk before the second auction, so the seller's opportunity cost at auction time is her value (zero). In the flower-grower example, this assumption means that the seller first produces the flowers and then offers them for sale without having a good option to sell them in some other market. When the seller uses a reserve price, she can instantaneously re-auction any unsold units. As a consequence of the sunk

\footnotetext{
${ }^{2}$ Either a third-party auctioneer keeps all the bids hidden and discloses only the price, or this is a Dutch auction.

${ }^{3}$ The cost-asymmetry between the two units simplifies the analysis by not modeling the decision whether or not to offer the first unit. The symmetric case of constant marginal cost is considered throughout as $c=0$.
} 
cost and instant re-auctioning, she can credibly use only a reserve equal to her value (zero) in either period (McAfee \& Vincent 1997). ${ }^{4}$ Note that even with these two reserve-weakening assumptions, ratcheting would lead to valuation-obscuring mixing strategies if second-price auctions were used instead of 1PSB: if the seller knows the highest remaining bidder has valuation exactly $v$, then take-it-or-leave-it offer at $v$ is credible (Levine \& Pesendorfer 1995).

Bidders: There are $N \geq 3$ unit-demand patient bidders indexed by $i$ with single-item valuations $x_{i}$ drawn iid from some continuous distribution $F$ on $[0,1]$. In the case of durables that provide a flow of benefits in each period, "valuation" is the net present value of the total utility derived from the good. There is no resale by the first-period winner to remaining bidders.

Information: The seller knows $F$, but $x_{i}$ are private information of each bidder. $c, \delta$ and $N$ are common knowledge at the start of the game, everyone learns $p_{1}$ in the end of the first period.

Selling regimes: Three selling regimes are considered: non-commitment, advance listing, and commitment to a first-period threshold price. The non-commitment seller plays a Perfect Bayesian Nash Equilibrium strategy. She decides whether or not to sell the second unit in the beginning of the second period after observing the first-period transaction price $p_{1}$. The seller who lists in advance decides her entire selling strategy in the beginning of the game. ${ }^{5}$ The seller with commitment to a threshold price can commit before the game to only produce and sell the second unit when $p_{1}$ weakly exceeds some cutoff level $m$ (for "minimum"). Both commitment sellers play a Nash equilibrium strategy.

Notation: Following Chapter 15 of Krishna (2002), let $Y_{k}^{(m)}$ be the $k$-th highest of $m$ draws drawn iid from $F$. Six important special cases used in this paper are: $X_{1} \equiv Y_{1}^{(N)} \quad X_{2} \equiv Y_{2}^{(N)}$ $X_{3} \equiv Y_{3}^{(N)} Y_{1} \equiv Y_{1}^{(N-1)} Y_{2} \equiv Y_{2}^{(N-1)} Z_{1} \equiv Y_{1}^{(N-2)}$. The distributions of the order-statistics of $N-1$ “competing" bidders are denoted $F_{j}(x)=\operatorname{Pr}\left(Y_{j} \leq x\right)$, with corresponding densities $f_{j}(x)$. The distributions of the order-statistics of the entire population of $N$ bidders are denoted $H_{j}(x)=\operatorname{Pr}\left(X_{j} \leq x\right)$, with corresponding densitites $h_{j}(x)$. It is convenient to also introduce $H_{2 \mid 1}(v \mid x)=\operatorname{Pr}\left(X_{2} \leq v \mid X_{1}=x\right)$, i.e. the conditional distribution of $X_{2}$ given $X_{1}$.

\footnotetext{
${ }^{4}$ The assumption of cost sunk before second auction together with instant re-auctioning make reserve-prices toothless. Section 4 investigates how changing these assumptions changes the seller's preferred strategy.

${ }^{5}$ The advance listing regime is revenue-equivalent to a simultaneous sale of both units via a third-price sealed-bid auction but delaying the delivery of the second unit. Please see Section 3.3 for details.
} 


\subsection{Bidding strategies}

The game can be solved by backward induction. I restrict attention to symmetric pure-strategy equilibria described by strictly increasing bidding strategies $\beta_{1}(x)$ and $\beta_{2}(x)$ in the first and second auction respectively. It will be shown that there exists a unique such equilibrium whenever $\delta$ is small enough.

Suppose the first-period bidding strategy $\beta_{1}(x)$ is pure and increasing. Then, the equilibrium second-auction bidding strategy $\beta_{2}(x)$ is standard because the first-period price does not impact second-period bids. This simplification is analogous to that in Milgrom \& Weber (2000), and it follows from mutual independence of the bidders: the first-period price reveals an upper bound $\beta_{1}^{-1}\left(p_{1}\right)$ on the valuations of the surviving bidders (i.e. the $N-1$ bidders who lose the first auction), but this bound does not bind survivors' optimization problem. The bound does not bind because each bidder only considers the remaining competition at or below his own valuation, and the revealed valuation $\beta_{1}^{-1}\left(p_{1}\right)$ of the first-period winner is by definition higher than that of all surviving bidders. Therefore, the surviving bidders bid according to the same function as bidders in a standard 1PSB with $N$-1 IPV bidders drawn from $F$ (see Krishna 2002 for a detailed proof):

Lemma 1: The unique symmetric second-period bidding strategy is:

$\beta_{2}(x)=\frac{1}{F^{N-2}(x)} \int_{0}^{x} v(N-2) f(v) F^{N-3}(v) d v=E\left(Z_{1} \mid Z_{1}<x\right)=E\left(Y_{2} \mid Y_{1}=x\right)=E\left(X_{3} \mid X_{2}=x\right)$

Unlike $\beta_{2}(x)$, first-period bidding depends on the seller's strategy. Because both noncommitment and advance listing correspond to special cases of a price-threshold, the following proposition characterizes bidding in all selling regimes (see the Appendix for all proofs):

Proposition 1: Let $m \in\left[0, E\left(Y_{1}\right)\right]$ be the seller's threshold price, and let $w$ be the bidder who would bid $m$ in the absence of the second auction: $m=E\left(Y_{1} \mid Y_{1}<w\right)$. When it is increasing, the following $\beta_{1}(x)$ is the unique increasing symmetric pure-strategy equilibrium:

$$
\beta_{1}(x)=E\left(Y_{1} \mid Y_{1}<x\right)-\delta \operatorname{Pr}\left(w<Y_{1} \mid Y_{1}<x\right) E\left(Y_{1}-Y_{2} \mid w<Y_{1}<x\right)
$$

When $\beta_{1}(x)$ is not increasing, there is no non-decreasing symmetric pure-strategy equilibrium. 
A key property of $\beta_{1}(x)$ is that bidders below $w$ bid as if there were no second auction while bidders above $w$ bid as if the second auction were guaranteed. The reason for the different expectations is that bidders correctly anticipate the seller's decision should they lose. When $x>w$, it is obvious that losing means that $p_{1}>m$ because losing means $Y_{1}>x$ and $x>w$. In contrast, a bidder with $x<w$ will only get to bid in the second auction when $Y_{1}>w$. Therefore, local deviations above $\beta_{1}(x)$ (slightly above $\beta_{1}(x)$ but still below $m$ ) do not change the chance that $Y_{1}>w$, and so low-value bidders do not consider the second-auction on the margin. Proposition 1 is surprising in that threshold-crossing deviations are not profitable either: even bidders slightly below $w$ do not find it beneficial to increase their bids in order to ensure the existence of the second auction. Such a deviation would only change the existence of the second auction if the bidder were pivotal to the outcome of the first auction, i.e. losing with the equilibrium bid but winning thanks to the deviation. But since winning the first auction rules out participation in the second, being pivotal implies that the deviation is not profitable.

Given that only bidders above $w$ shade their bids, it is possible to develop intuition for the shading decrement below the single-shot 1PSB strategy of $E\left(Y_{1} \mid Y_{1}<x\right)$ : in a first-price sealedbid auction, it is optimal to bid the expected valuation of the highest competitor conditional on winning the auction (Krishna 2002). A guaranteed existence of a future auction effectively reduces the valuation of the first unit by the opportunity cost of not winning the second unit - the discounted expected surplus $\delta\left[x-\beta_{2}(x)\right]$. Therefore, the valuation of the highest competing bidder net of the potential second-period auction is $Y_{1}-\mathbf{1}\left(Y_{1}>w\right) \delta\left[Y_{1}-\beta_{2}\left(Y_{1}\right)\right]$, from which the formula for $\beta_{1}(x)$ in Proposition 1 follows. One could therefore consider $\beta_{1}(x)$ to be an obvious candidate for the equilibrium bidding function. Proposition 1 confirms that this intuition indeed works by ruling out all possible deviations and establishing uniqueness.

The advance-listing and non-commitment special cases are as follows: When the seller lists in advance, no second sale ( $w=1$, hereafter called rationing) obviously yields standard 1PSB bidding $\beta_{1}^{0}(x)=E\left(Y_{1} \mid Y_{1}<x\right)$. Conversely, a guaranteed second sale $(w=0)$ yields the bid-shading strategy as in Milgrom \& Weber (2000), but modified for discounting:

$$
\beta_{1}^{1}(x)=\delta \beta_{1}^{M W}(x)+(1-\delta) \beta_{1}^{0}(x)=E\left(Y_{1} \mid Y_{1}<x\right)-\delta E\left(Y_{1}-Y_{2} \mid Y_{1}<x\right)
$$


where $\beta_{1}^{M W}(x)=E\left(Y_{2} \mid Y_{1}<x\right)$ is the original Milgrom \& Weber strategy. A non-commitment seller cannot pre-commit to an arbitrary $w$, and is instead bound by subgame perfection to select the breakeven $w_{0}$ that is implicitly defined by: $c=E\left(X_{3} \mid X_{1}=w_{0}\right)=\frac{1}{F^{N-1}\left(w_{0}\right)} \int_{0}^{w_{0}} \beta_{2}(v) d F^{N-1}(v)$.

\section{Figure 1: First-period bidding strategies (uniform example)}

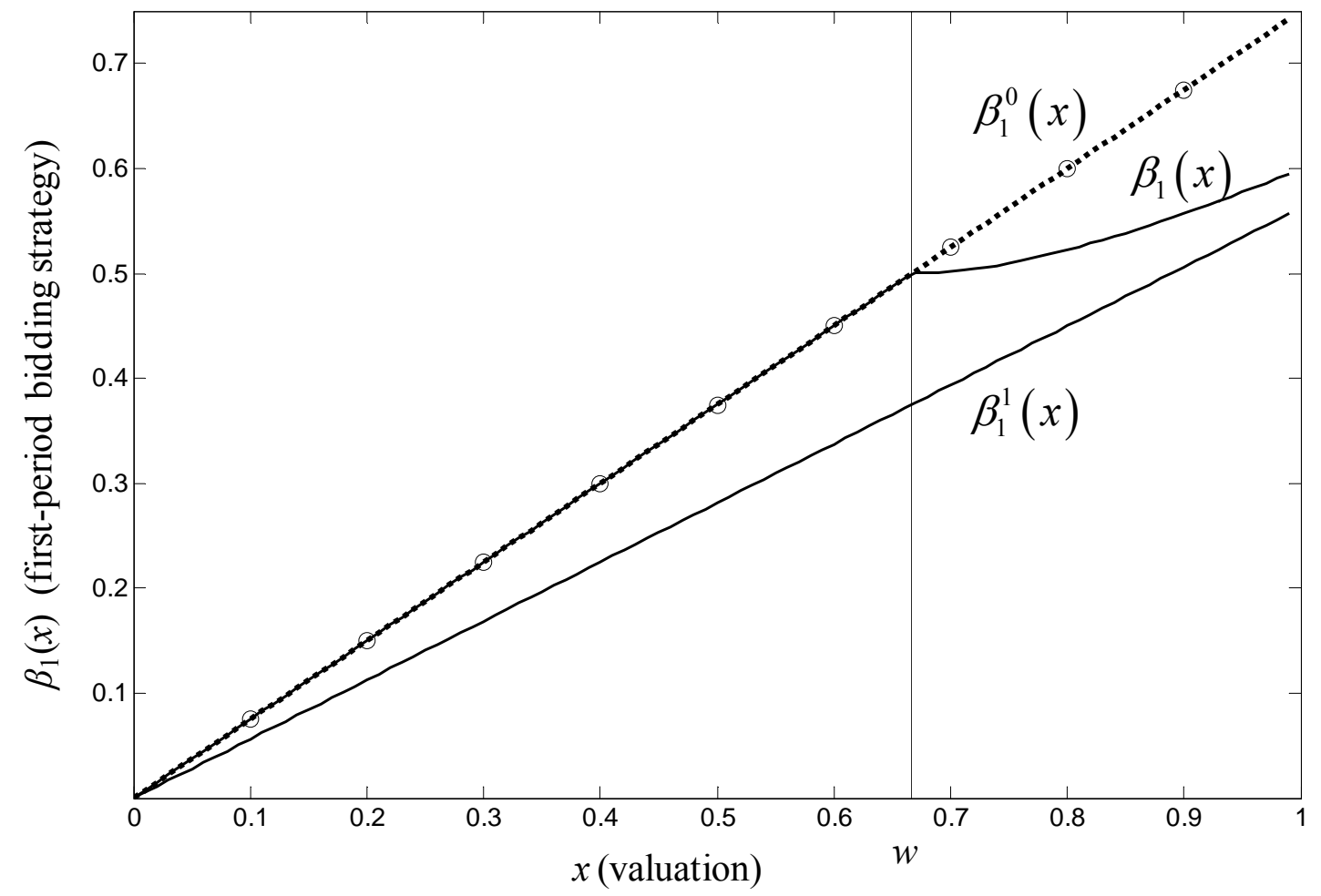

Note to Figure 1: The equilibrium first-period bidding strategy $\beta_{1}(x)$ is shown for the case of the Uniform distribution, $N=4$ bidders, $\delta=3 / 4$ discount factor, and $w=2 / 3$. The two strategies of the seller who lists in advance are also shown: the dashed line marked with circles shows the strategy when the seller rations, and the lowest solid line shows the strategy when the seller sells again for sure.

Example: When $F$ is a power distribution parametrized by $t>0$ as $F(x)=x^{t}$,

$$
\begin{aligned}
& \beta_{2}(x)=\frac{(N-2) t}{1+(N-2) t} x, \beta_{1}(x)=\frac{(N-1) t}{1+(N-1) t} x\left[1-\mathbf{1}(x>w) \delta \frac{x^{1+(N-1) t}-w^{1+(N-1) t}}{[1+(N-2) t] x^{1+(N-1) t}}\right], \text { and } \\
& w_{0}=\frac{[1+(N-2) t][1+(N-1) t]}{(N-2)(N-1) t^{2}} c . \text { When } t=1, F \text { is the uniform distribution, and the functions }
\end{aligned}
$$


simplify to $\beta_{2}(x)=\frac{N-2}{N-1} x, \beta_{1}(x)=\frac{x}{N}\left[N-1-\mathbf{1}(x>w) \delta \frac{x^{N}-w^{N}}{x^{N}}\right], \beta_{1}^{0}(x)=\frac{x}{N}(N-1)$ $>\beta_{1}^{1}(x)=\frac{x}{N}(N-1-\delta)$, and $w_{0}=\frac{c N}{N-2}$. Please see Figure 1 for an illustration.

One surprising aspect of Proposition 1 is that the existence of an invertible equilibrium is not guaranteed. Discounting of the second auction turns out to be necessary for existence:

Corollary 1: When $\delta=1$, there does not exist an increasing symmetric pure-strategy equilibrium for any $w \in(0,1)$. For the equilibrium to exist, $\delta$ needs to be small-enough. In the powerdistribution example, "small enough" $\delta$ is $\delta<\frac{1+(N-2) t}{1+(N-1) t}$.

To prove the Corollary, differentiate the candidate function $\beta_{1}(x)$. When $w \in\{0,1\}$, the slope of $\beta_{1}(x)$ is guaranteed to be positive. But when $0<w<1$, there is a kink at $w$ in that $\beta_{1}(x)$

becomes flatter at that point: $\left.\frac{d \beta_{1}}{d x}\right|_{x \neq w}=\frac{f_{1}(x)}{F_{1}(x)}\left[x-\beta_{1}(x)-\mathbf{1}(x>w) \delta\left(x-\beta_{2}(x)\right)\right]$

The reason for the kink is that marginal incentives change at $w$ : Below $w$, the marginal surplus of a winner with valuation $x$ is just the 1PSB surplus $x-\beta_{1}^{0}(x)$. Above $w$, however, winning means not participating in the second auction, and so the marginal surplus is $x-\beta_{1}(x)-\delta\left(x-\beta_{2}(x)\right)$. The kink at $w$ implies that the candidate function for $\beta_{1}(x)$ is not increasing for high $\delta$. When $\delta=1$, the right-hand slope at $w$ simplifies to:

$\lim _{x \rightarrow w+} \frac{d \beta_{1}}{d x}=\frac{f_{1}(w)}{F_{1}(w)}\left[\beta_{2}(w)-\beta_{1}^{0}(w)\right]<0$. This limiting slope is negative despite the fact that the derivative of $\beta_{1}^{1}(x)$ has a very similar form and is always positive:

$\frac{d \beta_{1}^{1}}{d x}=\frac{f_{1}(x)}{F_{1}(x)}\left[\beta_{2}(x)-\beta_{1}^{1}(x)\right]>0$ for all $x$. The reason for the different right-hand slopes of $\beta_{1}(x)$ and $\beta_{1}^{1}(x)$ at $w$ is the fact that under $\beta_{1}(x)$, low-value bidders with $x<w$ do not shade their bids below 1PSB levels, and so the equilibrium bid at $w$ is too high for $\beta_{1}(x)$ to keep increasing above $w$ when the second auction becomes a consideration and the incentives to shade 
"kick in". Specifically, $\delta=1$ sorts the three key bidding strategies as follows:

$\beta_{1}(w)=\beta_{1}^{0}(w)=E\left(Y_{1} \mid Y_{1}<w\right)>\beta_{2}(w)=E\left(Z_{1} \mid Z_{1}<w\right)>\beta_{1}^{1}(w)=E\left(\beta_{2}\left(Y_{1}\right) \mid Y_{1}<w\right)$.

It is not clear what happens when $\delta=1$, but Proposition 1 also shows that there cannot be a weakly increasing equilibrium with partial pooling at $m$, see the proof for details.

\subsection{Seller profits}

It is enough to evaluate the total expected seller profits in the price-threshold case. The total expected profit is the profit from the first auction plus the profit from the second auction whenever $X_{1}>w$. Thanks to the mutual independence of the bidders, the total expected profit can be expressed neatly in terms of order-statistics, as demonstrated by Proposition 2:

Proposition 2 : When the second unit is sold whenever $\beta_{1}^{-1}\left(p_{1}\right)>w$, total expected profits are:

$$
\Pi(w)=\Pi(0)+\delta\left\{\operatorname{Pr}\left(X_{2}<w\right) E\left(X_{2}-X_{3} \mid X_{2}<w\right)-\operatorname{Pr}\left(X_{1}<w\right) E\left(X_{3}-c \mid X_{1}<w\right)\right\}
$$

where $\Pi(0)=\Pi(1)+\delta\left[2 E\left(X_{3}\right)-E\left(X_{2}\right)-c\right]$ is the expected profit of a seller committed to hold the second auction, and where $\Pi(1)=E\left(X_{2}\right)$ is the expected profit of a rationing seller.

The first term in the curly brackets is the expected shading-reduction due to $w>0$, and the second term are the foregone second-period profits. Note that $\delta$ factors out of the curly bracket because expected shading is proportional to $\delta$. Therefore, the seller strategy does not depend on $\delta$ (as long as $\delta$ is small-enough to make $\beta_{1}(x)$ increasing). Also note that $E\left(X_{2}\right)$ is the baseline revenue in a standard 1PSB auction, which follows most easily by revenue equivalence of standard 1PSB with the second-price sealed-bid auction.

\subsection{Seller strategy: seller who lists in advance}

The simplest selling regime to discuss is the seller who lists in advance, i.e. the seller restricted to $w \in\{0,1\}$. Her optimal strategy is obvious from Proposition 2: she holds the second auction whenever $2 E\left(X_{3}\right)-E\left(X_{2}\right)>c$ and receives $\Pi(0)$, otherwise she rations the good and receives $\Pi(1)$. Note that compared to myopic 1PSB bidding, the first-period revenue when the seller lists the second unit in advance is reduced by $\delta\left[E\left(X_{2}\right)-E\left(X_{3}\right)\right]$ because of bid-shading. The bid- 
shading makes rationing more attractive than myopic bidding would, especially when the demand-side competition is low.

In the uniform example, the expectations of relevant order-statistics have a very simple form: $E\left(X_{2}\right)=\frac{N-1}{N+1}$ and $E\left(X_{3}\right)=\frac{N-2}{N+1}$. Therefore, the profit function simplifies to: $\Pi(0)=\frac{N-1}{N+1}+\delta\left(\frac{N-3}{N+1}-c\right)$, where the part in parentheses combines the additional secondperiod profit $\delta\left(\frac{N-2}{N+1}-c\right)$ with the profit-reduction due to shading of $\frac{\delta}{N+1}$. The seller thus prefers to ration whenever cost is high-enough, and there is always a region of the parameterspace, in which rationing is preferred to selling two units, namely $c>\frac{N-3}{N+1}$ (line MaxCommit in Figure 2). The above claim that bid-shading makes rationing more attractive than myopic bidding would can be illustrated as follows: when $N=3$, a seller facing $c>0$ will always ration, whereas the same seller would sometimes want to sell two units if bid-shading did not occur.

The advance listing regime is revenue-equivalent with allocating the two units via a single multi-unit auction. The natural auction to use would be a third-price sealed-bid auction:

Proposition 3: Suppose the seller uses a third-price sealed-bid auction to decide the allocation of both units in the first period, with the delivery of the second unit to the runner-up delayed until the second period. Then, the seller makes $\Pi(0)$, and the bidders follow a unique symmetric pure-strategy Nash equilibrium with a bidding function $\bar{\beta}(x)=\delta x+\frac{(1-\delta) x F(x)}{(N-2)[1-F(x)]}$. Because the runner up does not receive his full valuation at auction time, bidders do not have a dominant strategy to bid their valuations as they would in a canonical Vickrey auction. For the same reason, the standard multi-unit revenue-equivalence result of Maskin \& Riley (1989) does not apply either. Therefore, I prove this revenue equivalence directly, exposing an interesting bidding equilibrium in the process. For every $\delta<1, \bar{\beta}(x)$ is a peculiar bidding function for at least two reasons: First, high bidders $x$ such that $\frac{F(x)}{1-F(x)}>N-2$ bid more than their valuations. Second, $\bar{\beta}(x)$ approaches infinity as $x$ approaches the top of the valuation support. It 
is interesting how even a slight delay in delivery of the second unit completely changes the bidding incentives. Given the peculiarity of the bidding strategy, the third-price sealed-bid auction is not a practical way to sell the two units when the delivery of the second unit needs to be delayed due to production constraints assumed in this paper.

\subsection{Seller strategy: non-commitment seller}

The learning ability of the non-commitment seller makes her expected second-period profit positive, while also implying a cutoff $w_{0}>0$, which in turn implies a reduction in bid-shading in the first period relative to holding the second auction for sure. Therefore, the non-commitment seller always makes more than $\Pi(0)$. However, the non-commitment seller does not always outperform the seller who lists in advance because it is possible that $\Pi\left(w_{0}\right)<\Pi(1)$. While the expected non-commitment second-period profit is guaranteed to be positive, it may not compensate for first-period bid-shading. The following Proposition 4 characterizes when the non-commitment seller would find advance listing valuable:

Proposition 4 (Value of advance listing): The non-commitment seller would prefer commitment to advance listing whenever the bid-shading she faces in the first period exceeds the additional profit she expects to earn in the second period. This happens when

$$
E\left(X_{2}-X_{3} \mid X_{2}>w_{0}\right)>E\left(X_{3} \mid X_{2}>w_{0}\right)-E\left(X_{3} \mid X_{2}<w_{0}\right)
$$

In the uniform example, value of advance listing can be related explicitly to the parameters of the model because the formulae in Proposition 4 take on a neat form. It is obvious that the seller with $N>3$ and $c=0$ does not find advance listing valuable: she makes a second-period profit of $\delta\left(\frac{N-2}{N+1}\right)$ at the cost of only $\frac{\delta}{N+1}$ in added bid-shading. As the cost increases to the maximum feasible cost $\frac{N-2}{N}$ (line MaxLearn in Figure 2, i.e. cost such that $\left.w_{0}=1\right)$, both the additional profit and the additional bid-shading decrease all to way to zero - at which point the seller receives $\Pi(1)$. Since both components of additional profit decrease to zero, it is not immediately clear whether the shading decrement ever exceeds the additional profit for intermediate costs. The following Corollary 2 shows that this indeed happens, and it happens for high costs: 
Corollary 2: When $F$ is Uniform [0,1], then for all $N \geq 3$, there is a $c *(N) \leq \frac{N-3}{N-1}$ such that the non-commitment seller would strictly prefer to list in advance for every cost c such that $c^{*}(N)<c<\frac{N-2}{N} . c^{*}(N)$ increases in $N$.

Figure 2: Value of listing in advance relative to non-commitment (uniform example )

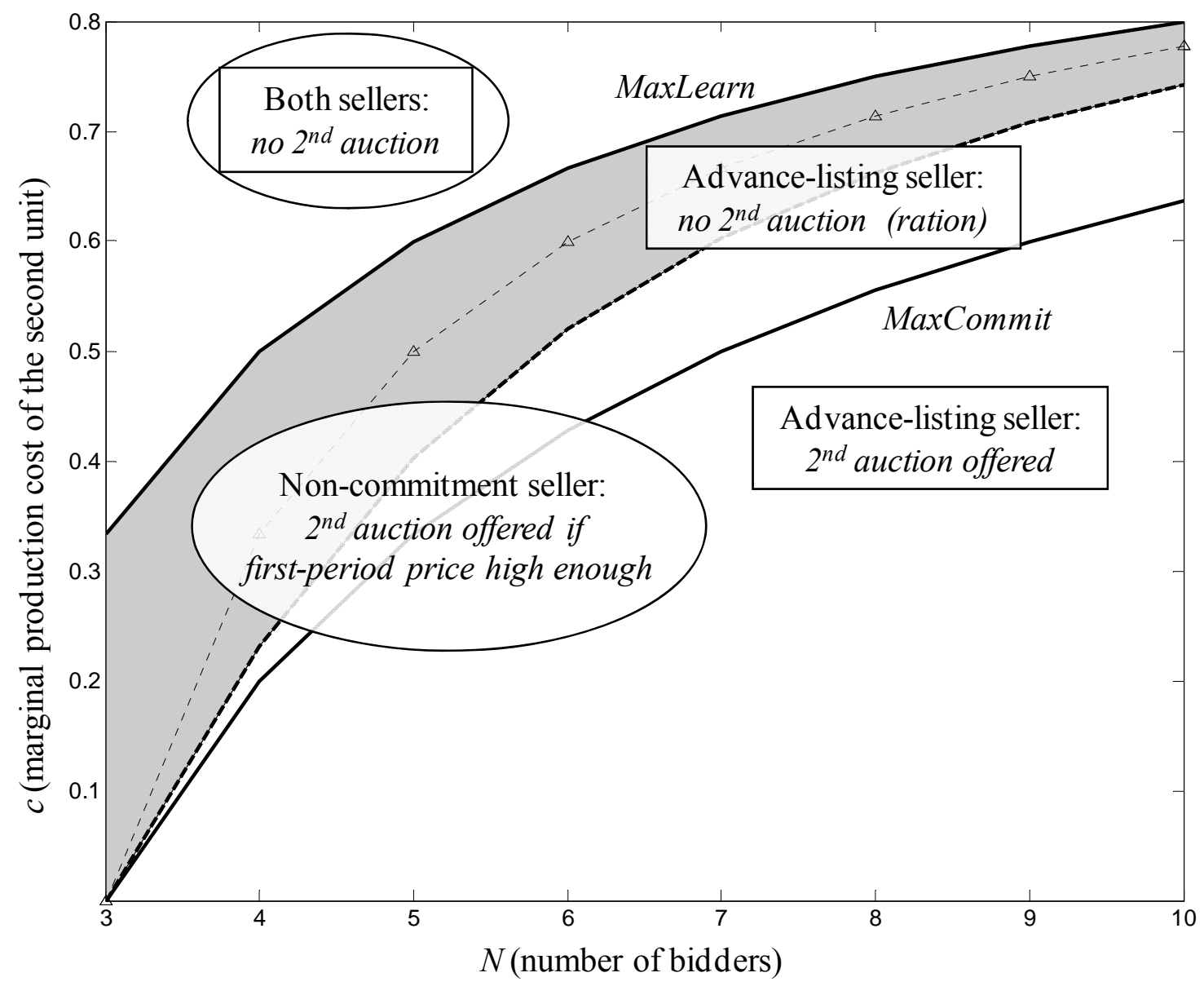

Note to Figure 2: The optimal non-commitment strategy is shown in ovals, optimal advance-listing strategy is shown in rectangles. Line MaxLearn is the maximum feasible cost of the second unit given first-period price, i.e. $(N-2) / N$. Line MaxCommit is the exante maximum feasible cost of the second unit, i.e. $(N-3) /(N+1)$. The shaded region is the region in which the non-commitment seller would strictly prefer listing in advance. The lower bound ary of the shaded region is $c^{*}(N)$ of Corollary 2. The thin dashed line marked with triangles is the analytical upper bound for $c^{*}(N)$ of Corollary 2 , i.e. $(N-3) /(N-1)$. The lines do not depend on $\delta$. 
Corollary 2 is illustrated in Figure 2. The fact that $c^{*}(N)$ increases in $N$ means that increased demand-side competition reduces the usefulness of advance listing relative to non-commitment selling. One way to interpret this result is as follows: Higher demand competition makes the second period more lucrative, so the non-commitment seller sells again more often ( $w_{0}$ decreases in $N$ ). Therefore, more bidders shade their bids, and so it may seem that the seller would be more interested in commitment to list in advance. However, the increased competition also reduces expected bidder surplus that drives bid-shading, and so the bidders shade by less. Corollary 2 shows that the second effect dominates the first, at least when $F$ is uniform.

\subsection{Seller strategy: seller with commitment to threshold price}

The seller who can commit to any $w$ solves the problem $\max _{w \in[0,1]} \Pi(w)$. As discussed above, this decision does not depend on $\delta$. From Proposition 2, the first-order condition is:

$$
h_{2}(w)\left[w-\beta_{2}(w)\right]=h_{1}(w) \int_{0}^{w}\left[\beta_{2}(v)-c\right] h_{2 \mid 1}(v \mid w) d v
$$

The LHS of the FOC is the marginal reduction in expected bid-shading, i.e. the bid-shading decrement of the bidder of type $w$. The RHS is the marginal increase in second-period profit, i.e. the expected profit $\beta_{2}\left(X_{2}\right)-c$ given that $X_{1}=w$. Loosely speaking, the FOC equates the marginal surplus to the bidders in the second auction with the marginal profit to the seller. The seller cares about the marginal second-auction surplus of the bidders because it enters firstperiod profits through bid-shading.

Solving the problem in general is difficult because the LHS margin is weighted by the $p d f$ of $X_{2}$ while the RHS margin is weighted by the $p d f$ of of $X_{1}$. However, it is easy to see that $w^{*}$ always exceeds $w_{0}$ because the RHS of the FOC is zero when $w=w_{0}$ and increasing above $w=w_{0}$. Therefore, whenever there exists a monotone pure-strategy equilibrium characterized in Proposition 1, commitment to threshold price strictly dominates non-commitment. Compared to the nuanced result of Proposition 4, the principle of commitment in dynamic games is thus restored when the commitment strategy is contingent on the observable first-auction outcome.

A general characterization of the optimal type-threshold $w^{*}$ is not feasible in closed form, but the uniform example yields a closed-form solution: When $F$ is Uniform[0,1], 
$h_{2 \mid 1}(v \mid w)=\frac{(N-1) v^{N-2}}{w^{N-1}}$, and so $w^{*}=\frac{N(1+c)}{2(N-1)}$ which in turn corresponds to the cutoff price of $m^{*}=\beta_{1}^{0}\left(w^{*}\right)=\frac{1+c}{2}$. Figure 1 shows the $w^{*}$ for the $N=4$ and $c=0$ case, along with the

associated $\beta_{1}(x)$. The surprising aspect of the uniform example is that $m^{*}$ does not depend on $N$ and corresponds exactly to the optimal reserve price in a single-shot 1PSB auction for the second unit, but with the cost $c$ not sunk until after the second auction. This match suggests that the two mechanisms may be equivalent on some level. The next section investigates this possibility, and shows that the above exact correspondence is a coincidence, and the two mechanisms are not equivalent.

\section{Commitment to a future reserve price}

In the threshold-price equilibrium, the highest bidder $X_{1}$ always receives a unit of the good, but the second-highest bidder $X_{2}$ is excluded from trading whenever $X_{1}$ is low (when $X_{1}<w^{*}$ ). The same exclusion could be accomplished by using a reserve price in the first period, and only producing the second unit if the first unit sells. If not (if no bids exceeded reserve), the first unit would be resold in the second period without a reserve. This mechanism would be less efficient than the threshold-price mechanism because it would delay the delivery of the unit to the highest bidder $X_{1}$. Moreover, Katsenos (2007) shows that bidding would be very complicated due to strategic abstentions of low-valuation bidders from the first auction.

Yet another way to exclude $X_{2}$ from trading is to use a second-period reserve price. The assumptions of Section 3 made second-period reserve prices weak by timing the production cost $c$ before the second auction, and by allowing the seller to instantaneously re-auction unsold units. Suppose instead that the cost does not have to be sunk until after the auction. In the flowergrower example, this cost-structure would arise if the grower only sold a promise to grow flowers after the second auction. ${ }^{6}$ In addition, suppose the seller can credibly commit not to reauction the second unit. To keep the amount of (inter-temporal) commitment the same as in the threshold-price case, suppose the seller can commit to any second-period reserve in the

\footnotetext{
${ }^{6}$ Another context with the cost not sunk until after the auction would be a seller endowed with two units and diminishing marginal utility: if I have two identical bicycles but I only ride one to work, I can sell one on eBay without reserve because it is worthless to me. If I get a really high price, I may want to sell my remaining bicycle as well, but my value of it is higher than that of my second bicycle.
} 
beginning of the game. It can be shown that under this alternative selling regime, a unique increasing symmetric pure-strategy equilibrium exists for all parameter values and all reserves. Interestingly, the optimal reserve to use turns out to be exactly the same as the reserve optimal for selling the second unit in isolation:

Proposition 5: Suppose there are $N \geq 3$ unit-demand bidders drawn iid from distribution $F$, such that $x-\frac{1-F(x)}{f(x)}$ is increasing in $x$. The seller sells one unit without a reserve, and pre-commits to a reserve price $r$ for a second unit sold in a future auction discounted by factor $\delta$. The seller's opportunity cost of the second unit at the time of the future auction is $c$. For every cost $c \geq 0$ and number of bidders $N \geq 3$, the r optimal for selling both units sequentially is the same as the optimal reserve price for selling only the second unit to the original group of bidders, namely $r^{*}$ such that $r^{*}=c+\frac{1-F\left(r^{*}\right)}{f\left(r^{*}\right)}$.

Two opposing forces act on the optimal reserve: increasing the second-period reserve obviously reduces bid-shading in the first period, so one might expect that the optimal second-period reserve for selling two units sequentially would be higher than the optimal reserve for selling the second unit in isolation. On the other hand, the first-unit sale also depletes the distribution of bidders by removing the highest one, and so the optimal reserve for the second-unit sale may actually be lower to accommodate the weaker demand. Proposition 5 shows that these two forces exactly cancel out.

It is possible to prove Proposition 5 by first deriving the equilibrium bidding strategy and then maximizing the implied seller profit. Instead, I use a direct proof that analyzes the equivalent direct revelation mechanism. ${ }^{7}$ The result extends earlier results from the mechanism design literature: Maskin \& Riley (1989) showed that the optimal direct revelation mechanism for selling many units to unit-demand bidders involves the same optimal reserve $r *$ for every unit as the direct revelation mechanism for selling just one unit in isolation. One implementation of the multi-unit optimal mechanism is a sequence of standard auctions which all have a reserve of $r^{*}$. Proposition 5 confirms that $r^{*}$ is ex-ante optimal for the second unit even when there is no first-period reserve.

\footnotetext{
${ }^{7}$ I thank an anonymous referee for suggesting the direct proof.
} 
A general revenue comparison of commitment to threshold price and commitment to a second-period reserve is beyond the scope of this paper. In the Uniform example, it can be shown that the second-period reserve revenue-dominates the threshold price, and it can also be shown that the commitment not to re-auction is necessary for this dominance. Therefore, even when the grower from the Introduction is only selling a promise to grow flowers after the second auction, she is better off with an optimal first-period price-threshold than with a second-period

reserve set at the production cost. Finally, the $m^{*}(c \mid N)=r^{*}(c)$ equality found in Section 3.4 for the Uniform case is a coincidence, because it does not hold in the more general powerdistribution case $F(x)=x^{t}$ (see Appendix for details of this example).

\section{Discussion}

An auction seller with the ability to procure additional units of a durable good in the future has a commitment problem because high-value buyers anticipate that should they lose today, the seller will find it profitable to produce and sell another unit. The high-value buyers therefore shade their bids down as if the future auction were guaranteed, reducing the seller's revenue from the current auction. Analogously to a durable-goods monopolist who sets prices, the seller may find it beneficial to credibly announce that she will not sell another unit in the future. However, this posted-price market intuition is not complete because a canonical auction seller starts out uncertain about demand and has a natural ability to learn about demand from the outcomes of early auctions. Therefore, the auction seller may want to make future auctions contingent on the outcome of the current auction. This paper presents a model of a seller who makes the future auction contingent on today's price reaching a threshold.

A key technical contribution of this paper is a general analysis of the bidding strategy induced by a price threshold. No invertible (increasing symmetric pure-strategy) equilibria exist for the sequential second-price sealed-bid auction because of ratcheting: the auction would reveal the valuation of the highest surviving bidder, and the seller would exploit that bidder by setting the second-period reserve-price equal to that valuation. An invertible bidding equilibrium does exist for the Dutch auction (i.e. a first-price sealed-bid auction with only the price revealed), but only when the second auction is sufficiently discounted by the bidders, for example when it is delayed. When it exists, the first-period bidding strategy is qualitatively different for low and high bidders: the low bidders bid as if there were no chance of a future auction, while the high 
bidders bid as is the future auction were guaranteed. Because of this difference, the equilibrium bidding strategy has a kink in that it becomes locally flatter as it exceeds the threshold price. When the second auction occurs instantaneously after the first auction, no informative equilibrium exists even for the Dutch auction, nor does an equilibrium with partial pooling. A mixed-strategy equilibrium occurs, and the seller thus cannot interpret the price as the bid of the highest bidder.

When an informative equilibrium does not exist, or when the commitment needed for the credibility of an arbitrary threshold price is not available, the seller may be able to ration the good, i.e. commit not to sell the future unit under any circumstances. Commitment to ration may be more realistic as it is naturally available to all sellers in markets that require advance listing of future auctions. By rationing the good, the seller concentrates the demand-side competition in the first auction but foregoes the future revenue completely. When she lists a future auction, she suffers from first-period bid-shading by all bidders but gets the future profit (which may actually be negative if her opportunity cost is high). Either way, and regardless of discounting, there exists a unique symmetric pure-strategy bidding equilibrium. When the future auction is delayed, the simple bidding equilibrium in the sequential auction for two units contrasts with relatively non-intuitive strategies that would arise if the seller wanted to allocate both units through the revenue-equivalent third-price sealed-bid auction (Proposition 3).

Schelling's commitment principle in dynamic games implies that commitment to a threshold price always dominates non-commitment. In contrast, commitment to advance listing is too coarse to always outperform non-commitment. A key question thus arises: when would a seller prefer advance listing (no learning) to non-commitment (with learning)? The regime preference between advance listing and non-commitment boils down a production decision at the margin, and so it is natural for the seller to consider marginal revenue versus marginal cost. One contribution of this paper is to highlight the composition of these marginals: First, because the bidders are strategic and forward-looking, the correct marginal revenue to consider must be net of the reduction in first-period revenue due to bid-shading. Second, the correct marginal cost is the cost of production minus the information rent from non-commitment's better-informed production decision. The indifference condition of the seller can be written in terms of conditional order-statistics (Proposition 4), and analyzed explicitly under the uniform distributional assumption (Corollary 2). An interesting result emerges in the uniform special 
case: advance listing is preferred when the cost is high and demand weak in the sense of a small number of bidders. As the demand strengthens, advance listing is less and less useful. The reason for the latter effect is that increased demand-side competition reduces the bidder surplus from the second auction, in turn reducing the incentive to shade early bids in a non-commitment auction. While the main focus of this paper is the threshold-price model, I also considered two other forms of intertemporal commitment to a contingent future strategy. First, the seller could use a reserve price in the first period, re-auction the first unit if no bids exceed it, and only produce the second unit if the first unit sells. This mechanism would allocate the unit(s) to exactly the same bidder(s) as the threshold price, but the delivery of the first unit to the highest bidder would sometimes be delayed until the second period. Because of the same allocation but the additional delay, it is unlikely that the seller could make a higher profit through this mechanism. Another way the seller could reduce future supply would be to pre-commit to a future reserve price. This mechanism would work well if the seller could commit not to reauction unsold units. The optimal reserve price to use is surprisingly easy to characterize: it is the same reserve the seller would use to auction only the second unit in isolation (Proposition 5). Therefore, unlike the optimal threshold price, the optimal future reserve does not depend on the number of bidders. The seller's relative preference between the two contingent strategies depends on the timing of production costs: the first-period threshold can always consider the second-period production cost on the margin, while a second-period reserve can only consider it if it is not sunk before the second auction. As a result, first-period threshold-prices can dominate second-period reserves in terms of overall profit. 


\section{Appendix: Proofs of propositions}

\section{Proof of Proposition 1}

Proof: For any function $\beta_{1}(x)$ to constitute a symmetric-equilibrium bidding function, there must be no profitable deviations from $\beta_{1}(x)$ for any $x \in[0,1]$. This proof first uses necessary firstorder conditions for $x \neq w$ to derive a unique continuous candidate function, confirms that the candidate function offers no profitable deviations to any $x \in[0,1]$, and shows that continuity at $w$ is necessary for equilibrium.

Suppose $\beta_{1}(x)$ is strictly increasing so first-period bids have the same ordering as valuations. It is helpful to introduce notation $B(x)$ for "baseline" ex-ante expected surplus from the second auction: $B(x)>0$ because the following three events can co-occur for every $x: 1$ ) $Y_{1}>x$ and $x$ thus loses the first auction 2) $Y_{1} \geq w$ and so the second auction is offered by the seller 3) $Y_{2}<x$ and so $x$ wins the second auction. $B(x)$ captures the resulting expected surplus: $B(x) \equiv \delta \operatorname{Pr}\left[Y_{1}>\max (w, x) \& Y_{2}<x\right]\left[x-\beta_{2}(x)\right]=$ $=\delta(N-1)[1-F(\max (w, x))] F^{N-2}(x)\left[x-\beta_{2}(x)\right]=\delta(N-1)[1-F(\max (w, x))] \int_{0}^{x} F^{N-2}(v) d v$ The first equality evaluates the joint probability of 1) to 3 ), i.e. the probability that there is one competing bidder above $\max (w, x)$ and $N-2$ competing bidders below $x$ (noting that the highest competing bidder can be selected $N-1$ different ways). The third equality follows from Lemma 1 $\&$ integration by parts, which together imply $F^{N-2}(x)\left[x-\beta_{2}(x)\right]=\int_{0}^{x} F^{N-2}(v) d v$. The latter is a familiar result: the expected surplus at the start of an IPV sealed-bid auction is the integrated probability of winning the auction. Hereafter, I denote $S_{2}(x) \equiv \int_{0}^{x} F^{N-2}(v) d v$.

Consider a bidder with $x \neq w$ who bids $\beta_{1}(z)$ instead of $\beta_{1}(x)$, but only deviates locally in that $|z-x|<|w-x|$. There are two cases: CASE 1: For $x<w$, the local profit function is the same as that in a 1PSB except there is the second-period surplus $B(x)$ :

$\Pi(z \mid x, z<w)=\left[x-\beta_{1}(z)\right] F_{1}(z)+B(x)$. Since $B(x)$ is constant in $z$, the local maximum of $\Pi$ must satisfy the same first-order condition (FOC) as a standard bidding strategy in a single 1PSB: $\frac{d \beta_{1}(z) F_{1}(z)}{d z}=x f_{1}(z)$. Symmetry of the equilibrium then implies that the FOC must hold with $x=z$ so that type $x$ finds it locally optimal to bid $\beta_{1}(x)$. Therefore, for $x<w$, the $\beta_{1}(x)$ must satisfy the differential equation $\frac{d \beta_{1}(x) F_{1}(x)}{d x}=x f_{1}(x)$ with the initial condition $\beta_{1}(0)=0$. 
CASE 2: For $x>w$, the profit function is the same as in Milgrom \& Weber (2000) except that the second-period surplus is discounted. There are two sub-cases depending on the direction of the local deviation from $x$ :

$$
\begin{aligned}
& \Pi(z \mid x, w<x<z)=\left[x-\beta_{1}(z)\right] F_{1}(z)-\delta[F(z)-F(x)]\left[x-\beta_{2}(x)\right](N-1) F^{N-2}(x)+B(x) \\
& \Pi(z \mid x, w<z<x)=\left[x-\beta_{1}(z)\right] F_{1}(z)+\delta \int_{z}^{x}\left[x-\beta_{2}\left(y_{1}\right)\right] f_{1}\left(y_{1}\right) d y_{1}+B(x)
\end{aligned}
$$

where $z<x$ deviation is more involved because losing to $y_{1} \in(z, x)$ implies that it is optimal to bid only $\beta_{2}\left(y_{1}\right)<\beta_{2}(x)$ in the second period (see Krishna 2002). The FOC in each subcase coincide at $z=x$ and imply the differential equation $\frac{d \beta_{1}(x) F_{1}(x)}{d x}=\left[x(1-\delta)+\delta \beta_{2}(x)\right] f_{1}(x)$

The above FOC determine the slopes of any candidate $\beta_{1}(x)$ at $x \neq w$, i.e. the slope of any $\beta_{1}(x)$ that offers no local deviations at $x \neq w$. It is immediate from the FOC that any candidate must be continuous and differentiable at $x \neq w$. Moreover, there is a unique way to connect the $x<w$ and $x>w$ regions to achieve continuity at $w$, namely using $\lim _{x \rightarrow w_{-}} \beta_{1}(x \mid x<w)$ as the initial condition for the differential equation describing the $x>w$ region:

$$
\begin{aligned}
\beta_{1}(x) & =\frac{1}{F_{1}(x)} \int_{0}^{x}\left[v-\delta 1(v>w)\left(v-\beta_{2}(v)\right)\right] d F_{1}(v)= \\
& =\frac{1}{F_{1}(x)} \int_{0}^{x} v d F_{1}(v)-\mathbf{1}(x>w) \frac{\delta(N-1)}{F_{1}(x)} \int_{w}^{x} S_{2}(v) f(v) d v= \\
& =E\left[Y_{1} \mid Y_{1}<x\right]-\delta \operatorname{Pr}\left(w<Y_{1} \mid Y_{1}<x\right) E\left[Y_{1}-Y_{2} \mid w<Y_{1}<x\right]
\end{aligned}
$$

where the second equation follows from $S_{2}(x)=F^{N-2}(x)\left[x-\beta_{2}(x)\right]$, and the third equation follows from multiplying and dividing the first equation by $\operatorname{Pr}\left(w<Y_{1}<x\right)$.

I now show that $\beta_{1}(x)$ offers no deviations $\beta_{1}(z)$ to any bidder $x \in[0,1]$. There are six different orderings of $x, z$, and $w$. The cases $z<x \leq w$ and $x<z<w$ are the same as in standard 1PSB, (see Krishna, p. 18). The cases $w \leq x<z$ and $w \leq z<x$ are straightforward generalizations of the Milgrom \& Weber result, and I include them here for intuition, completeness, and as building-blocks for the subsequent evaluation of threshold-crossing deviations:

$$
\begin{aligned}
& \Pi(z \mid x, w \leq x<z)=x F_{1}(z)-\int_{0}^{z} v d F_{1}(v)+\delta(N-1) \int_{w}^{z} S_{2}(v) d F(v)-\delta(N-1) S_{2}(x) \int_{x}^{z} d F(v)+B(x) \\
& \Rightarrow \Pi(x \mid x, w \leq x<z)-\Pi(z \mid x, w \leq x<z)=\delta(N-1) \int_{x}^{z}\left[(v-x) F^{N-2}(v)-\int_{x}^{v} F^{N-2}(y) d y\right] d F(v) \geq 0
\end{aligned}
$$

The intuition behind the result is as follows: in a single-shot 1PSB, $\Pi(z \mid x)=x F_{1}(z)-\int_{0}^{z} v d F_{1}(v)$, which is maximized at $z=x$. From the the first two terms of the first line, it is thus evident that by bidding $\beta_{1}(z)>\beta_{1}(x)$, the bidder loses as if by bidding too much in a single-shot 1PSB. At the 
same time, he also gets a net gain because the additional expected price-reduction due to bidshading (third term) exceeds the foregone expected continuation payoff (fourth term). As the second-line shows, however, the net gain will always be smaller than the loss from overbidding in a single-shot 1PSB. The case of $w \leq z<x$ is analogous, with the intuition reversed:

$$
\begin{aligned}
& \Pi(z \mid x, w \leq z<x)=x F_{1}(z)-\int_{0}^{z} v d F_{1}(v)+\delta(N-1) \int_{w}^{z} S_{2}(v) d F(v)+\delta \int_{z}^{x}\left[x-\beta_{2}(v)\right] d F_{1}(v)+B(x) \\
& \Rightarrow \Pi(x \mid x, w \leq z<x)-\Pi(z \mid x, w \leq z<x)=(1-\delta) \int_{z}^{x}(x-v) d F_{1}(v) \geq 0
\end{aligned}
$$

The remaining two cases treat the threshold-crossing deviations, namely $x<w \leq z$ and $z<w \leq x . \Pi(z \mid x, x<w \leq z)$ is the same as $\Pi(z \mid x, w \leq x<z)$ except that the last integral (the fourth term) is from $w$ to $z$ instead from $x$ to $z$ : the continuation payoff is only available if the highest opponent is above $w$. The net profit from deviation to $\beta_{1}(z)$ decomposes as follows:

$$
\Pi(x \mid x, x<w \leq z)-\Pi(z \mid x, x<w \leq z)=[\Pi(w \mid x=w<z)-\Pi(z \mid x=w<z)]+\int_{x}^{w}(v-x) d F_{1}(v) \geq 0
$$

where the part in brackets was shown to be positive above (in the $w \leq x<z$ case), and the remaining integral just $\Pi(x \mid x<w)-\Pi(w \mid x<w)$ in the single-shot 1PSB case. Intuitively, a threshold-crossing deviation from $x<w$ to $z>w$ yields the same profit as the same deviation would to a bidder with $w=x$ minus the additional loss from overbidding in the first auction.

Finally, the $z<w \leq x$ deviation is also not profitable because the deviation to $z=w$ is not profitable by above argument in the $w \leq z<x$ case, and further reduction in $z$ below $w$ yields the same net surplus as bidder with $x=w$ deviating to $z<w$ :

$$
\Pi(x \mid x, z<w<x)-\Pi(z \mid x, z<w<x)=[\Pi(x \mid w<x)-\Pi(w \mid w<x)]+\int_{z}^{w}(x-v) d F_{1}(v) \geq 0
$$

It remains to be shown that continuity of $\beta_{1}(x)$ at $w$ is necessary for equilibrium.

Suppose not, and suppose there is a jump $J>0$ such that $\tilde{\beta}_{1}(x)=\beta_{1}(x)+\delta \mathbf{1}(x>w) J$. Then, there is a type $w+\varepsilon$ that will find it profitable to deviate to $w$. Plugging the new $\tilde{\beta}_{1}(x)$ candidate into the $w \leq z<x$ case analyzed above yields $\Pi(w \mid x=w+\varepsilon)-\Pi(x \mid x=w+\varepsilon)=J-(1-\delta) \int_{w}^{w+\varepsilon}(x-v) d F_{1}(v)$, and so there is an $\varepsilon>0$ such that the deviation is profitable. Alternatively, consider the other possible jump of $\tilde{\beta}_{1}(x)=\beta_{1}(x)+\delta \mathbf{1}(x \geq w) J$. Then, plugging the new $\tilde{\beta}_{1}(x)$ into the $z<w \leq x$ case above yields $\Pi(w-\varepsilon \mid x=w)-\Pi(w \mid x=w)=J-\int_{w-\varepsilon}^{w}(x-v) d F_{1}(v)$, and so there is a type $w$ - $\varepsilon$ such that $w$ will deviate to $w-\varepsilon$. This concludes the proof that when $\beta_{1}(x)$ is increasing, it defines a unique symmetric pure-strategy equilibrium.

When $\beta_{1}(x)$ is not increasing, there obviously does not exist an increasing equilibrium. There is also is no weakly-increasing pure-strategy equilibrium, i.e. an equilibrium with partial pooling at $m$. Suppose otherwise, and assume all opponents bid according to a weakly-increasing 
$\beta_{1}(x)$ such that $\beta_{1}(x)=m$ for all $x \in[\underline{w}, \bar{w}]$. Now consider a focal bidder with $x \in[\underline{w}, \bar{w}]$, and let $\tau$ be the probability of the highest opponent(s) bidding $m$ and the resulting tie-break resulting in a win of the focal bidder. The focal bidder's best response to the opponents is almost never to bid $m$ : by bidding $m$, he gets expected surplus $\left[F_{1}(\underline{w})+\tau\right](x-m)+\delta(1-\tau)\left(x-\beta_{2}(x)\right)$. Now consider two epsilon deviations: bidding $m-\varepsilon$ avoids the tie at $m$ by always losing the first auction instead, and profit changes by $\Delta P \equiv+\tau\left[\delta\left(x-\beta_{2}(x)\right)-(x-m)\right]$. Alternatively, bidding $m+\varepsilon$ avoids the tie by always winning instead and the profit changes by $-\Delta P$. Since $\beta_{2}(x)$ is increasing, it is impossible for $\Delta P$ to be zero for all $x \in[\underline{w}, \bar{w}]$, and so one of the epsilon deviations is almost always profitable. Therefore $\beta_{1}(x)$ not increasing rules out all nondecreasing symmetric pure-strategy equilibria. $Q E D$

\section{Proof of Proposition 2}

Let $d G(v, x) \equiv d H_{211}(v \mid x) d H_{1}(x)$, i.e. the differential of the joint distribution of the top two order-statistics. Also, denote the profit from $w=0$ as $\Pi(0)=E\left(X_{2}\right)+\delta E\left(2 X_{3}-X_{2}-c\right)$. Then, the profit from setting $w \in[0,1]$ is:

$$
\begin{aligned}
\Pi(w) & =E_{X_{1}}\left[\beta_{1}\left(X_{1}\right)\right]+\delta \operatorname{Pr}\left(X_{1}>w\right) E_{X_{1} \mid X_{1}>w}\left[E_{X_{2} \mid X_{1}}\left(\beta_{2}\left(X_{2}\right)-c\right)\right]= \\
= & \int_{0}^{1} \int_{0}^{x} v d G(v, x)-\delta \int_{w}^{1} \int_{w}^{x}\left[v-\beta_{2}(v)\right] d G(v, x)+\delta \int_{w}^{1} \int_{0}^{x}\left[\beta_{2}(v)-c\right] d G(v, x)
\end{aligned}
$$

where the first term corresponds to standard 1PSB revenue $E\left(X_{2}\right)$, the second term captures the shading in the first period, and the last term captures the second-period profits.

Now add and subtract the shading and second-period profit of the case $w=0$ :

$$
\begin{aligned}
& =E\left(X_{2}\right)-\delta \int_{0}^{1} \int_{0}^{x}\left[v-\beta_{2}(v)\right] d G(v, x)+\delta \int_{0}^{1} \int_{0}^{x}\left[\beta_{2}(v)-c\right] d G(v, x) \\
& +\delta \int_{0}^{w} \int_{0}^{x}\left[v-\beta_{2}(v)\right] d G(v, x)+\delta \int_{w}^{1} \int_{0}^{w}\left[v-\beta_{2}(v)\right] d G(v, x)-\delta \int_{0}^{w} \int_{0}^{x}\left[\beta_{2}(v)-c\right] d G(v, x)
\end{aligned}
$$

Noting that $h_{2}(v)=N(N-1) f(v) F^{N-2}(v)[1-F(v)]$, change integration order:

$$
\begin{aligned}
& \int_{0}^{u} \int_{0}^{x} v d G(v, x)+\int_{u}^{1} \int_{0}^{u} v d G(v, x)=\int_{0}^{u} v h_{2}(v) d v=\operatorname{Pr}\left(X_{2}<u\right) E\left(X_{2} \mid X_{2}<u\right) \\
& \text { Analogously, } \int_{0}^{u} \int_{0}^{x} \beta_{2}(v) d G(v, x)+\int_{u}^{1} \int_{0}^{u} \beta_{2}(v) d G(v, x)=\int_{0}^{u} \beta_{2}(v) h_{2}(v) d v=\operatorname{Pr}\left(X_{2}<u\right) E\left(X_{3} \mid X_{2}<u\right)
\end{aligned}
$$
and $\int_{0}^{u} \int_{0}^{x} \beta_{2}(v) d G(v, x)=\operatorname{Pr}\left(X_{1}<u\right) E\left(X_{3} \mid X_{1}<u\right)$. Therefore, for any $w \in[0,1]$ : 


$$
\begin{aligned}
\Pi(w) & =\Pi(0)+\delta\left\{\int_{0}^{w}\left[v-\beta_{2}(v)\right] h_{2}(v) d v-\int_{0}^{w} \int_{0}^{x}\left[\beta_{2}(v)-c\right] h_{2 \mid 1}(v \mid x) d v h_{1}(x) d x\right\}= \\
& =\Pi(0)+\delta\left\{\operatorname{Pr}\left(X_{2}<w\right) E\left(X_{2}-X_{3} \mid X_{2}<w\right)-\operatorname{Pr}\left(X_{1}<w\right) E\left(X_{3}-c \mid X_{1}<w\right)\right\} \quad Q E D
\end{aligned}
$$

\section{Proof of Proposition 3}

Suppose there is an increasing function $\beta(x)$ that all the $N-1$ opponents follow. A bidder with valuation $x$ who pretends to be $z$ gets surplus:

$$
\begin{aligned}
\Pi(z ; x) & =x \operatorname{Pr}\left(Y_{1}<z\right)+\delta x \operatorname{Pr}\left(Y_{2}<z<Y_{1}\right)-\operatorname{Pr}\left(Y_{2}<z\right) E\left[\beta\left(Y_{2}\right) \mid Y_{2}<z\right]= \\
& =\int_{0}^{z}[x-\beta(y)] d F_{2}(y)-x(1-\delta)(N-1) F^{N-2}(z)[1-F(z)]
\end{aligned}
$$

where the first term is the standard third-price sealed-bid auction surplus, and the second term reflects the reduction in surplus due to the delay in receiving the good whenever $z$ is below the top but still above the second highest competitor. The $F O C$ is:

$$
\text { FOC : }[x-\beta(z)] f_{2}(z)=x(1-\delta)(N-1) f(z) F^{N-3}(z)[N-2-(N-1) F(z)]
$$

In a symmetric equilibrium, FOC must hold with $z=x: \beta(x)=\delta x+\frac{(1-\delta) x F(x)}{(N-2)[1-F(x)]}$

The expected revenue of the seller is: $2 E_{X_{3}}\left[\beta\left(X_{3}\right)\right]=2 E_{X_{2}}\left[E_{X_{3} \mid X_{2}}\left[\beta\left(X_{3}\right)\right]\right]=$

$$
\begin{aligned}
& =2 \int_{0}^{1} \int_{0}^{x_{2}}\left[\delta x_{3}+(1-\delta) x_{3}\left(\frac{F\left(x_{3}\right)}{(N-2)\left[1-F\left(x_{3}\right)\right]}\right)\right] \frac{d F^{N-2}\left(x_{3}\right)}{F^{N-2}\left(x_{2}\right)} d H_{2}\left(x_{2}\right)= \\
& =2 \delta E\left(X_{3}\right)+2(1-\delta) \int_{0}^{1} \int_{0}^{x_{2}} x_{3} \frac{F\left(x_{3}\right)}{\left[1-F\left(x_{3}\right)\right]} f\left(x_{3}\right) F^{N-3}\left(x_{3}\right) d x_{3} N(N-1) f\left(x_{2}\right)\left[1-F\left(x_{2}\right)\right] d x_{2}= \\
& =2 \delta E\left(X_{3}\right)+(1-\delta) \int_{0}^{1} x_{3} N(N-1) f\left(x_{3}\right) F^{N-2}\left(x_{3}\right)\left[1-F\left(x_{3}\right)\right] d x_{3}=\Pi(0)+\delta c
\end{aligned}
$$

because $\int_{x_{3}}^{1} f\left(x_{2}\right)\left[1-F\left(x_{2}\right)\right] d x_{2}=\left[1-F\left(x_{3}\right)\right]\left[\frac{1-F\left(x_{3}\right)}{2}\right]$ by parts. $Q E D$

\section{Proof of Proposition 4}

For any $w$, the expected profit in Proposition 2 can be transformed:

$$
\begin{aligned}
\Pi(w) & =\Pi(1)-\delta \int_{w}^{1} \int_{w}^{x}\left[v-\beta_{2}(v)\right] d G(v, x)+\delta \int_{w}^{1} \int_{w}^{x}\left[\beta_{2}(v)-c\right] d G(v, x)+\delta \int_{w}^{1} \int_{0}^{w}\left[\beta_{2}(v)-c\right] d G(v, x)= \\
& =\Pi(1)+\delta\left\{\operatorname{Pr}\left(X_{2}>w\right) E\left(2 X_{3}-X_{2}-c \mid X_{2}>w\right)+\operatorname{Pr}\left(X_{1}>w>X_{2}\right) E\left(X_{3}-c \mid w>X_{2}\right)\right\}
\end{aligned}
$$

Noting that $E\left(X_{3}-c \mid w_{0}>X_{2}\right)=E\left(X_{3}-c \mid X_{1}=w_{0}\right)=0$, the non-commitment seller makes: $\Pi\left(w_{0}\right)=\Pi(1)+\delta \operatorname{Pr}\left(X_{2}>w_{0}\right) E\left(2 X_{3}-X_{2}-c \mid X_{2}>w_{0}\right)$. Finally, $c$ can be expressed in terms of $w_{0}$ because $c=E\left(X_{3} \mid X_{1}=w_{0}\right)=E\left(X_{3} \mid X_{2}<w_{0}\right) \cdot Q E D$ 


\section{Proof of Corollary 2}

First express $c$ in terms of $w_{0}$ :

$$
\begin{aligned}
& \Pi\left(w_{0}\right)<\Pi(1) \Leftrightarrow \int_{w_{0}}^{1} \int_{w_{0}}^{x}\left[\beta_{2}(v)-\frac{N-2}{N} w_{0}\right](N-1) v^{(N-2)} d v N d x<\int_{w_{0}}^{1} \int_{w_{0}}^{x}\left[v-\beta_{2}(v)\right](N-1) v^{(N-2)} d v N d x \\
& \Leftrightarrow \text { additional profit }=\left(\frac{N-2}{N(N+1)}\right)\left[N-(N+1) w_{0}+w_{0}^{N+1}\right]<\frac{1-(N+1) w_{0}^{N}+N w_{0}^{N+1}}{N+1}=\text { additional shading } \\
& \Leftrightarrow \lambda\left(w_{0}\right)=-N(N-3)+(N-2)(N+1) w_{0}-N(N+1) w_{0}^{N}+\left(N^{2}-N+2\right) w_{0}^{N+1}>0
\end{aligned}
$$

When $N=3, \lambda(w)>0$ for every $w \in(0,1)$, and $\lambda(0)=\lambda(1)=0$, so the seller prefers advance listing for every $c>0$ and is indifferent when $c=0$. When $N \geq 4$, the $\lambda(w)$ has the following properties: $\lambda(0)<0, \lambda^{\prime}(0)>0, \lambda^{\prime \prime}(0)=0, \lambda(1)=0, \lambda^{\prime}(1)=0, \lambda^{\prime \prime}(1)=2 N(N+1)>0$ Since $\lambda^{\prime \prime}(1)>0$ and $\lambda^{\prime}(1)=\lambda(1)=0$, there exists a $w<1$ such that $\lambda(w)>0$ and $\lambda^{\prime}(w)<0$. Together with the fact that $\lambda(0)<0$, the intermediate value theorem applied to $\lambda$ implies that there is another $z<w, z \in(0,1)$ such that $\lambda(z)=0$. The intermediate value theorem applied to $\lambda^{\prime}$ then implies that there is a $v \in(z, 1)$ such that $\lambda^{\prime}(v)=0$. This $v$ must be the only root of $\lambda^{\prime}$ in $(0,1)$ because: $\lambda^{\prime}(w)=(N-2)(N+1)-N^{2}(N+1) w^{N-1}+(N+1)\left(N^{2}-N+2\right) w^{N}$ and so $\lambda^{\prime}(w)=0 \Leftrightarrow \frac{N-2}{N^{2}}=\frac{(1-w) w^{N-1}}{1-w^{N}}$, where LHS is a constant and RHS is strictly increasing in $w$ : $\frac{d}{d w}\left(\frac{(1-w) w^{N-1}}{1-w^{N}}\right)>0 \Leftrightarrow N>\frac{1-w^{N}}{1-w}=\sum_{k=0}^{N-1} w^{k}$ which is true for every $N$ and $w \in(0,1)$. Since $v$ is the unique root of $\lambda^{\prime}$ in $(0,1), z$ is the unique root of $\lambda$ in $(0,1)$, and $\lambda$ is negative below $z$ (seller prefers advance listing) and positive above $z$ (seller prefers non-commitment). The cutoff cost is obtained from the definition of $w_{0}: c *(N)=\frac{N-2}{N} z$. The sufficient bound $c^{*}(N)<\frac{N-3}{N-1}$ follows from a region in which shading exceeds profit not just in expectation but for all values of $X_{2}>w_{0}: \beta_{2}(v)-\frac{N-2}{N} w_{0}<v-\beta_{2}(v) \Leftrightarrow \frac{(N-2)(N-1)}{N(N-3)} w_{0}>1 \Leftrightarrow c<\frac{N-3}{N-1}$. It remains to be shown that $c^{*}(N)$ increases in $N$. Since $\frac{N-2}{N}$ increases in $N$, it is sufficient to prove that wherever $\lambda(w)$ is increasing, increasing $N$ reduces $\lambda(w)$, and so $\lambda(w \mid N)$ intersects the $x$-axis further to the right from $\lambda(w \mid N-1)$. The claim is: $\lambda^{\prime}(w \mid N)>0 \Rightarrow \Delta \lambda(w) \equiv[\lambda(w \mid N)-\lambda(w \mid N-1)]<0$. To prove the claim, note $\Delta \lambda(w)=4-2 N+2(N-1) w+N(N-1) w^{N-1}-\left(N^{2}-N+2\right) w^{N}(2-w)$, add and subtract $2 w^{N-1}$ to obtain: $\Delta \lambda(w)=2\left(1-w^{N-1}\right)-2(N-1)(1-w)-(N-2) w^{N-1}(1-w)^{2}+N^{2} w^{N-1}(1-w)(1-w)$ 
From above, $\lambda^{\prime}(w \mid N)>0 \Rightarrow N^{2} w^{N-1}(1-w)>(N-2)\left(1-w^{N}\right)$, so

$$
\begin{aligned}
& \Delta \lambda(w)<2\left(1-w^{N-1}\right)-2(N-1)(1-w)-(N-2) w^{N-1}(1-w)^{2}+(N-2)\left(1-w^{N}\right)(1-w)= \\
& =N w\left(1-w^{N-2}\right)-(N-2)\left(1-w^{N}\right) \equiv \mu(w)<0
\end{aligned}
$$

To see the last inequality, note that $\mu(0)<0, \mu(1)=0, \mu^{\prime}(0)>0, \mu^{\prime}(1)=0$, and $\mu^{\prime \prime}(w)<0$ in $(0,1)$, so $w=1$ is the only root of $\mu(w)$ in $[0,1]$, ensuring that $\mu(w)<0$ in $(0,1) \cdot Q E D$

\section{Proof of Proposition 5}

The sequential auction with pre-commitment to a second-period reserve is revenue equivalent with the direct-revelation mechanism that allocates one unit of the good to the highest bidder immediately, and one unit to the second-highest bidder in the future. Because the bidders are risk-neutral, the delay of the second unit enters their utility in the same way as a reduction in probability, so the probability of type- $x$ bidder winning a unit in the equivalent direct revelation mechanism is: $q(x)=F^{N-1}(x)+\delta(N-1)[1-F(x)] F^{N-2}(x) \mathbf{1}(x>r)$.

Since $q(x)$ is non-decreasing in $x$, standard incentive-compatibility and individual-rationality arguments (Myerson 1981) can be used to derive the expected payment $m(x)$ of a single bidder:

$$
E_{x}[m(x)]=\int_{0}^{1}\left[x q(x)-\int_{0}^{t} q(t) d t\right] f(x) d x=E_{x}\left[\left(x-\frac{1-F(x)}{f(x)}\right) q(x)\right]
$$

Because the bidders are drawn iid from $F$, the expected revenue from $N$ bidders is simply $N E_{x}[m(x)]$. The expected cost is just $\delta c \operatorname{Pr}\left(X_{2}>r\right)$, which can be written as: $\delta c \operatorname{Pr}\left(X_{2}>r\right)=N E_{x}\left[\delta c(N-1)(1-F(x)) F^{N-2}(x) \mathbf{1}(x>r)\right]$. Substituting $q(x)$ into the expected revenue and subtracting the expected cost yields the expected profit:

$$
\Pi(r)=N E_{x}\left[\left(x-\frac{1-F(x)}{f(x)}\right) F^{N-1}(x)+\delta(N-1)(1-F(x)) F^{N-2}(x) \mathbf{1}(x>r)\left(x-c-\frac{1-F(x)}{f(x)}\right)\right]
$$

The direct revelation mechanism thus additively separates the impact of $r$ on $\Pi(r)$ into the effect of screening the second highest bidder, irrespective of the other bidders. Since $x-\frac{1-F(x)}{f(x)}$ is increasing, it is immediate that screening at $r$ such that $\left(r-c-\frac{1-F(r)}{f(r)}\right)=0$ is optimal. $Q E D$ 


\section{Power-distribution example of relationship between $m^{*}$ and $r^{*}$ :}

Claim: When $F(x)=x^{t}$ on $[0,1]$ with $t>0$, there is a unique threshold price $m^{*}(c \mid N, t)$ for every $N$ and $t$. Let $r *(c \mid t)$ be the optimal second-unit reserve price. For every $N$, $m^{*}(c \mid N, t)>r^{*}(c \mid t)$ when $t>1$, and $m^{*}(c \mid N, t)<r^{*}(c \mid t)$ when $t<1$. Proof: $F(x)=x^{t}$ implies $h_{2 \mid 1}(v \mid w)=\frac{t(N-1) v^{t(N-1)-1}}{w^{t(N-1)}}$, and so the FOC of the $\max _{w \in[0,1]} \Pi(w)$ problem is: $\frac{N-1}{1+(N-2) t}=\left[\frac{(t+1)(N-1)}{1+(N-1) t} w-c\right] w^{t-1}$. The LHS of the equation is a positive quantity constant in $w$, and the RHS of the equation is positive and increasing for $1>w>c \frac{1+(N-1) t}{(t+1)(N-1)}$. By the intermediate value theorem, there is a unique $w^{*}$ that solves the equation, and the implied $m^{*}$ is $m^{*}=\beta_{1}\left(w^{*}\right)=\frac{(N-1) t}{1+(N-1) t} w^{*}$. The optimal reserve-price $r$ satisfies $c=r-\frac{1-F(r)}{f(r)}\left(\right.$ Proposition 5) which becomes $(t+1) r^{t}-c t r^{t-1}=1$ under the $F(x)=x^{t}$ assumption. Inverting the $\beta_{1}$ at the optimal reserve by using the bidding function form $r=\frac{(N-1) t}{1+(N-1) t} w_{r}$ yields the optimal-reserve condition in terms of the cutoff type $w_{r}$ : $\frac{1}{t}\left(\frac{1+(N-1) t}{(N-1) t}\right)^{t-1}=\left[\frac{(t+1)(N-1)}{1+(N-1) t} w_{r}-c\right] w_{r}^{t-1}$. Note that the RHS of the optimal-reserve condition is the same as the RHS of the FOC equation above, so $w_{r}>w^{*}$ whenever $\frac{1}{t}\left(\frac{1+(N-1) t}{(N-1) t}\right)^{t-1}>\frac{N-1}{1+(N-2) t}$ and vice versa. This inequality holds iff: $\lambda(t) \equiv t \log [t(N-1)]-\log [1+(N-2) t]-(t-1) \log [1+(N-1) t]<0$. Since $\lambda(1)=0$ and $\lambda^{\prime}(t)>0$ for all $t \geq 1, t>1 \Rightarrow \lambda(t)>0$. Since $\lambda(0) \equiv \lim _{t \rightarrow 0+} \lambda(t)=0$ and $\lim _{t \rightarrow 0+} \lambda^{\prime}(t)=-\infty, \lambda(t)<0$ near zero. Since $\lambda(1)=0$ and $\lambda^{\prime}(1)>0,0<t<1 \Rightarrow \lambda(t)<0$ unless $\lambda$ has a root in $(0,1)$. Such a root does not exist because it would imply at least two roots of $\lambda "$ in $(0,1)$. However, $\lambda "(t)=0 \Leftrightarrow 1-t-N(N-2) t^{2}=0$, and so there is only one positive root of $\lambda "(t)$, namely $\frac{-1+\sqrt{1+4 N(N-2)}}{2 N(N-2)}<1$. So, 1 is the unique positive root of $\lambda$, and $0<t<1 \Rightarrow \lambda(t)<0 Q E D$ 


\section{References}

Caillaud, B. and Mezzetti, C. (2004). Equilibrium Reserve Prices in Sequential Ascending Auctions. Journal of Economic Theory 117(1), 78-95.

Coase, R. H. (1972). Durability and Monopoly. Journal of Law and Economics 15, 143-149.

Engelbrecht-Wiggans, R. (1994). Sequential Auctions of Stochastically Equivalent Objects. Economics Letters 44, 87-90.

Freixas, X., Guesnerie, R. and Tirole, J. (1985). Planning under Incomplete Information and the Ratchet Effect. Review of Economic Studies 52(2), 173-91.

Jeitschko, T. D. (1999). Equilibrium price paths in sequential auctions with stochastic supply, Economics Letters 64, 67-72.

Katsenos, G. (2007). Optimal reserve prices in sequential auctions with imperfect commitment. Chapter 2, PhD Thesis, University of Pittsburgh.

Krishna, V. (2002). Auction Theory. Academic Press of Elsevier Science, London, UK.

Levine, D.K. and Pesendorfer, W. When are Agents Negligible? American Economic Review $85(5), 1160-1170$.

Maskin, E. and Riley, J. (1989). Optimal Multi-unit Auctions, in Frack Hahn (Ed.), The Economics of Missing Markets, Information, and Games. Oxford University Press, UK.

McAfee, R.P. and Vincent, D. (1997). Sequentially Optimal Auctions. Games and Economic Behavior 18, 246-276.

Milgrom, P. R. and Weber, R.J. (2000). A Theory of Auctions and Competitive Bidding II. Paul Klemperer, ed. The Economic Theory of Auctions, Edward Elgar Publishing.

Myerson, R. (1981). Optimal Auction Design. Mathematics of Operations Research 6, 58-73.

Stokey, Nancy. (1979). Intertemporal Price Discrimination. Quarterly Journal of Economics 93, 355-371.

Skreta, V. (2006). Sequentially Optimal Mechanisms. Review of Economic Studies 73, 10851111.

Skreta, V. (2007) Optimal Auction Design under Non-Commitment. Working paper. New York University.

Zeithammer, R. (2007). Optimal Selling in Sequential Auctions: Commitment vs. Adaptation. Marketing Science 26 (6), 859-867. 